\title{
All regular Landsberg metrics are Berwald
}

\section{Zoltán Imre Szabó}

Received: 26 September 2007 / Accepted: 19 March 2008 / Published online: 9 April 2008

(C) Springer Science+Business Media B.V. 2008

\begin{abstract}
This article negatively answers the long standing problem concerning the existence of non-Berwald Landsberg metrics.
\end{abstract}

Keywords Finsler geometry $\cdot$ Landsberg metrics $\cdot$ Berwald metrics

\section{Introduction}

Finsler metrics generalize the Riemann metrics like the Banach spaces do the Hilbert spaces. In Riemann geometry the norms of tangent vectors $y \in T_{p}\left(M^{n}\right)$ at a fixed point $p \in$ $M^{n}$ are Euclidean, while in Finsler geometry these norms, defined by appropriate Finsler functions $L_{p}(y)$, are Minkowskian. On proper Finsler manifolds, these functions define proper Riemann metric tensors $g_{i j}(p, y)=(1 / 2) \partial_{y^{i}} \partial_{y^{j}}\left(L_{p}^{2}\right)$ on the punctured tangent spaces $T_{p}(M) \backslash 0$. The Berwald connections generalize the Riemann connections, which, however, do not satisfy all properties of their Riemannian ancestors. For instance, the Berwald parallel transports just keep the norms of the tangent vectors, but the displacements, $\tau_{c}: T_{p}\left(M^{n}\right) \rightarrow$ $T_{q}\left(M^{n}\right)$, of the tangent spaces along curves $c$ are neither linear nor isometries regarding the metrics $g_{i j}$ in general.

The Finsler metrics having linear Berwald connections are called Berwald metrics, and those having isometric Berwald parallel transports are called Landsberg metrics. It is well known that all Berwald metrics are Landsberg. The question if this inclusion is proper? has been one of the longest standing open problems in Finsler geometry. Matsumoto had called it, in 2004, the most important unsolved question of the field. Due to the many unsuccessful

Communicated by: H.-B. Rademacher (Leipzig).

Z. I. Szabó (凶)

Lehman College of CUNY, Bronx, NY 10468, USA

e-mail: zoltan.szabo@lehman.cuny.edu

Z. I. Szabó

Rényi Institute, PO Box 127, Budapest 1364, Hungary 
attempts made for finding non-Berwald Landsberg metrics, D. Bao calls them "unicorns"; beautiful creatures who have never been seen by human beings [5]. Matsumoto's call strongly amplified these researches, but only examples which are Landsberg just on the complement of certain singularity-subsets of the tangent bundle $[3,4,11]$, or, generalized unicorns $[5,8]$ appeared on the horizon. These constructions are not in the scope of this article. Detailed review of these results can be found in the expository article [6].

In this note we prove that regular Landsberg metrics are always Berwald. In other words, regular "Landsberg-unicorns" do not exist. The proof of this statement is based on the following very simple idea: For a given Landsberg metric one constructs, first, a Riemannian metric tensor $\mathbf{g}_{i j}(p)$ by integrating the Landsberg metric tensor $g_{i j}(p, y)$ on the unit balls $B_{p} \subset T_{p}\left(M^{n}\right)$ by the measure $\mu_{p}=\sqrt{\operatorname{det}\left(g_{i j}\right)(p, y)} d y^{1} \wedge \cdots \wedge d y^{n}$. Since the unit balls are invariant under the action of the Berwald parallel transports, furthermore, the Berwald covariant derivative $\nabla_{k}$ of a Landsberg space satisfies the relations $\nabla_{k} g_{i j}=0$ and $\nabla_{k} \mu=0$, also the relation $\nabla_{k} \mathbf{g}_{i j}=0$ must hold. This means that the torsion free Berwald connection must be the Levi Civita connection of the Riemannian metric $\mathbf{g}_{i j}(p)$. That is the Berwald connection is linear, indeed.

It should be mentioned that, in the classification theorems established in $[12,13]$, the author corresponded Riemann metrics also to Berwald metrics. The linear Berwald connections become the Levi Civita connections of the corresponded Riemann metrics also in those cases. However, those Riemann metrics were constructed in a different way, namely, by using the holonomy groups of the linear Berwald connections. On Berwald manifolds, the construction introduced in this article provides Riemann metrics which can be constructed also by the holonomy groups. However, the technique of holonomy groups does not apply to Landsberg metrics.

\section{Berwald connections and Berwald metrics}

In the following, the base manifold and its tangent bundle are denoted by $M^{n}$ and $T\left(M^{n}\right)$, respectively. A coordinate system $\mathbf{x}=\left(x_{1}, \ldots, x_{n}\right)$ defined on $M^{n}$ induces a coordinate system, $(\mathbf{x}, \mathbf{y})=\left(x_{1}, \ldots, x_{n}, y_{1}, \ldots, y_{n}\right)$, on the tangent bundle. Although several objects will be described in terms of such coordinates, the following considerations have global features.

The norms of tangent vectors are defined by the Finsler function $L(\mathbf{x}, \mathbf{y})$, satisfying the following regularity conditions:

(1) It is positively homogeneous, meaning $L(\mathbf{x}, \lambda \mathbf{y})=\lambda L(\mathbf{x}, \mathbf{y}), \forall \lambda>0$.

(2) The convexity assumption requires that the metric tensor field $g_{i j}=\frac{1}{2} \partial_{y_{i}} \partial_{y_{j}}\left(L^{2}\right)$, defined on the punctured tangent space $T\left(M^{n}\right) \backslash 0$, must be smooth and positive definite.

(3) The theorems established in this article are valid for metric tensor $g_{i j}(\mathbf{x}, \mathbf{y})$ of class $C^{2}$. An equivalent assumption is that the Finsler function $L(\mathbf{x}, \mathbf{y})$ must be of class $C^{4}$.

Note that, for any fixed $\mathbf{x} \in M^{n}$, the $g_{i j}(\mathbf{x}, \mathbf{y})$ defines a priori non-Euclidean Riemann metric on $T_{\mathbf{x}}\left(M^{n}\right) \backslash 0_{\mathbf{x}}$. This metric is Euclidean, for all $\mathbf{x}$, exactly for Riemann metrics.

The most important object what should be generalized to Finsler manifolds is the Riemann connection and the associated parallel transports along curves running on $M^{n}$. A basic requirement is that the arc-wise parameterized geodesics should appear as auto-parallel curves also on Finsler manifolds. This generalized object was established by Berwald. In modern terminology, it is called non-linear connection defined on the tangent bundle. Speaking in abstract, it is a smooth positively homogeneous distribution, $H(\mathbf{x}, \mathbf{y}) \subset T_{(\mathbf{x}, \mathbf{y})}\left(T\left(M^{n}\right)\right)$, of $n$-dimensional horizontal subspaces which are complement to the natural vertical subspaces 
$T_{(\mathbf{x}, \mathbf{y})}^{v}\left(T\left(M^{n}\right)\right)$. The latter subspaces consist of vectors tangent to curves running in $T_{\mathbf{x}}\left(M^{n}\right)$ through $(\mathbf{x}, \mathbf{y})$. Such a distribution is spanned by local vector fields $\partial_{x^{i}}^{H}=\partial_{x^{i}}-G_{i}^{r}(\mathbf{x}, \mathbf{y}) \partial_{y^{r}}$, which are the horizontal lifts of fields $\partial_{x^{i}}$ (defined on $M^{n}$ ) to the tangent bundle. These formulas can be regarded as definitions for the symbols $G_{i}^{r}(\mathbf{x}, \mathbf{y})$ of a non-linear connection as well. For an arbitrary smooth curve $\mathbf{x}(t):[a, b] \rightarrow M$ and a vector $X(\mathbf{x}(a)) \in T_{\mathbf{x}(a)}\left(M^{n}\right)$, there is a unique extension, $X(\mathbf{x}(t)) \in T_{\mathbf{x}(t)}\left(M^{n}\right)$, of $X(\mathbf{x}(a))$ onto $\mathbf{x}(t)$ such that the curve $\left(\mathbf{x}(t), X(\mathbf{x}(t)):[a, b] \rightarrow T\left(M^{n}\right)\right.$ is horizontal. The corresponding differential equation describing this extension in terms of the component functions $\mathbf{x}(t)=\left(x_{1}(t), \ldots, x_{n}(t)\right)$ and $X(\mathbf{x}(t))=X^{i}(t) \partial_{x^{i}}(t)$ is

$$
\frac{d X^{i}}{d t}(t)+\frac{d x^{r}}{d t}(t) G_{r}^{i}(\mathbf{x}(t), X(t))=0 .
$$

The diffeomorphism $\tau_{\mathbf{x}(a) \mathbf{x}(b)}: T_{\mathbf{x}(a)}(M) \rightarrow T_{\mathbf{x}(b)}(M)$ defined by the correspondence $X(\mathbf{x}(a)) \rightarrow X(\mathbf{x}(b))$ is called parallel transport along $\mathbf{x}(t)$. The covariant derivative from this parallel transport is denoted by $\nabla_{k}$, or, $\mid k$. For a covariant Finsler tensor field $\mu$, it is traditionally defined by the $\operatorname{limit}_{\dot{\mathbf{x}}(a)} \mu=\lim _{b \rightarrow a}\left(\tau_{\mathbf{x}(a) \mathbf{x}(b)}^{*}\left(\mu_{\mathbf{x}(b)}\right)-\mu_{\mathbf{x}(a)}\right) /(b-a)$. This definition requires a so called vertical representation of the Finsler tensor fields. For instance, the metric tensor should be considered in the form $g_{i j} d y^{i} \otimes d y^{j}$.

Berwald observed that, for any Finsler manifold $\left(M^{n}, L\right)$, there is a unique positively homogeneous non-linear connection satisfying the metric conditions $\partial_{x^{k}}^{H}(L)=L_{\mid k}=0$ and having torsion free symbols $G_{i j}^{k}:=\partial_{y^{i}} G_{j}^{k}$, i.e., satisfying $T_{i j}^{k}:=G_{i j}^{k}-G_{j i}^{k}=0$. In fact, by the latter equation, $G_{i}^{k}=(1 / 2) \partial_{y^{i}}\left(G^{k}\right)$ must hold, where $G^{k}:=y^{j} G_{j}^{k}$. Furthermore, let the operator $y^{k} \partial_{y^{i}}$ act on both sides of equations $L L_{\mid k}=0$ to see that the sought non-linear connection has the uniquely determined symbols

$$
G^{i}=\frac{g^{r i}}{4}\left(y^{k}\left(L^{2}\right)_{x^{k} y^{r}}-\left(L^{2}\right)_{x^{r}}\right) .
$$

On Riemann manifolds, the objects $G_{i j}^{k}:=\partial_{y^{i}} G_{j}^{k}$ depend just on $\mathbf{x}$ and are nothing but the Christoffel symbols of the linear Levi Civita connections, which are uniquely determined by the identities $T=0$ and $g_{i j \mid k}=0$. By the above computations, however, the Levi Civita connections of Riemann manifolds are uniquely determined by the three properties:

$$
\begin{array}{rlrl}
(A): & G_{l}^{i}(\mathbf{x}, \lambda \mathbf{y})=\lambda G_{l}^{i}(\mathbf{x}, \mathbf{y}), & \forall \lambda>0, \\
(B): \quad T_{i j}^{k}=G_{i j}^{k}-G_{j i}^{k}=0, \quad(C): & L_{\mid k}=0,
\end{array}
$$

not just among the linear but also the much larger class of non-linear connections, which a priori define positively homogeneous but non-linear parallel transports. Also note that the metric condition (C) ensures only that the Berwald parallel transports keep the norm of the tangent vectors, but, for a general Finsler manifold, the diffeomorphisms $\tau_{\mathbf{x}(a) \mathbf{x}(b)}: T_{\mathbf{x}(a)}(M) \rightarrow$ $T_{\mathbf{x}(b)}(M)$ are not isometries with respect to the Riemann metrics defined by $g_{i j} d y^{i} \otimes d y^{j}$ on $T_{\mathbf{x}(a)}\left(M^{n}\right) \backslash 0$ and $T_{\mathbf{x}(b)}(M) \backslash 0$, respectively. Thus we have:

Theorem 2.1 For a Finsler manifold $\left(M^{n}, L\right)$, there is a unique Berwald connection satisfying the three characteristic properties described in (3). In terms of the Finsler function, the symbols of this connection are described in (2).

If a non-linear connection satisfies properties $(A)$ and $(B)$, furthermore, $(C)$ is satisfied for two Finsler functions such that one of them is Riemann, then the connection is linear (namely, it is the Levi Civita connection of the Riemann metric) and the other metric is Berwald. In local terminology these conditions mean that the symbols (2) for the two metrics 
are the same, thus also the Christoffel symbols $\Gamma_{j k}^{i}$ of the Riemann metric are the same as the Berwald symbols $G_{j k}^{i}$ belonging to the other Finsler metric.

Berwald metrics are defined by those Finsler metrics for which the Berwald connections are still linear. These metrics are constructed and explicitly determined, by several classification theorems, in $[12,13]$. These metrics are "perturbed" Cartesian products of irreducible Riemann spaces and such non-Riemannian Berwald metrics which are constructed on irreducible symmetric manifolds of rank $>1$. The latter irreducible factor-manifolds are explicitly determined by means of the Chevalley's polynomials. The results include the complete determination of the isometry groups acting on these manifolds. Also the Cartan-symmetric Finsler manifolds, defined by those Finsler metrics for which the geodesic involutions are isometries, are completely determined. It turns out, that all these metrics are Berwald, thus the classification of Berwald metrics provides classification also for these latter symmetric metrics.

Let it be mentioned yet that these constructions start out by a theorem asserting that the linear Berwald connection of a Berwald metric is always Riemann metrizable, i.e., there exists a Riemann metric whose Levi Civita connection is the considered linear Berwald connection. That Riemannian metric is constructed by the Haar measure defined on the holonomy group of the linear connection. Although in a slightly different way, the same idea is used in this article. Now, the Riemann metric $\mathbf{g}_{i j}(\mathbf{x})$ corresponded to a Landsberg metric $g_{i j}(\mathbf{x}, \mathbf{y})$ is defined by the integral of $g_{i j}$ on the unit ball $B_{\mathbf{x}} \subset T_{\mathbf{x}}(M)$ with respect to the measure defined by the $g_{i j}$. It turns out that also this Riemann metric is parallel (i.e., $\mathbf{g}_{i j \mid k}=0$ holds) with respect to the Berwald connection of the Landsberg metric. Thus, by Theorem 2.1, the Landsberg metric must be Berwald. It should be mentioned yet that on Berwald manifolds this construction corresponds Riemann metrics which can be constructed also by means of holonomy groups.

\section{Landsberg metrics}

There is pointed out above that the parallel transports $\tau_{\mathbf{x}(a) \mathbf{x}(b)}: T_{p}(M) \rightarrow T_{q}(M)$ are a priori not isomeries with respect to the Riemann metrics defined by $g_{i j}=\frac{1}{2} \partial_{y_{i}} \partial_{y_{j}}\left(L^{2}\right)$ on the tangent spaces $T_{p}(M) \backslash 0$ and $T_{q}(M) \backslash 0$. The Landsberg metrics are defined by those Finsler metrics where these transports are isometries. In terms of the Berwald covariant derivative, this property is equivalent to $g_{i j \mid k}=0$.

The only examples known for Landsberg metrics are Berwald, for which the Landsberg property can be proved by the well known equation

$$
L l_{r} G_{i j k}^{r}=g_{i j \mid k}, \text { where } G_{i j k}^{r}=\partial_{y^{i}} G_{j k}^{r} .
$$

The left side of (4) obviously vanishes on Berwald manifolds. (To see this identity, take out $\partial_{y^{i}} \partial_{y^{j}}$ on the left side, first. Then, apply the metric condition: $\frac{1}{2} \partial_{x^{k}}\left(L^{2}\right)=L l_{r} G_{k}^{r}$ before taking them back, which is the last step in proving the equation. This statement is established also in [6].)

Let it be mentioned yet that the Landsberg property is usually defined $[7,9,10]$ by the identity $L l_{r} G_{i j k}^{r}:=\dot{A}_{i j k}=0$. Note that this definition requires Finsler function of class $C^{5}$. Under this assumption of smoothness, formula (4) establishes the equivalence of this condition with $g_{i j \mid k}=0$. Also note that, by (2), the latter condition requires metric tensor being only of class $C^{2}$, or, Finsler function of class $C^{4}$. Under these conditions the field $g_{i j \mid k}$ is continuous.

Next we prove the inclusion in the opposite direction: 
Theorem 3.1 Regular Landsberg metrics having metric tensors $g_{i j}$ of class $C^{2}$, or Finsler function of class $C^{4}$, are always Berwald.

Proof This statement is established by constructing a Riemannian metric, $\mathbf{g}$, which is also parallel regarding the Berwald connection of the considered Landsberg metric, i.e., the metric condition $\mathbf{g}_{i j \mid k}=0$ holds. Thus, by Theorem 2.1, the Berwald connection is linear, which must be equal to the Levi Civita connection of the Riemann metric $\mathbf{g}$.

For a fixed $\mathbf{x} \in M$, the Riemann metric $g_{i j}(\mathbf{x}, \mathbf{y})$ non-trivially depends on the tangent vectors $\mathbf{y} \in T_{\mathbf{x}}(M) \backslash 0$ in general. The sought Riemann metric tensor, $\mathbf{g}_{i j}(\mathbf{x})$, at $\mathbf{x}$ is constructed by integrating $g_{i j}(\mathbf{x}, \mathbf{y})$ on the unit ball $B_{\mathbf{X}}=\left\{\mathbf{y}_{\mathbf{x}} \in T_{\mathbf{x}}(M) \mid L\left(\mathbf{x}, \mathbf{y}_{\mathbf{x}}\right) \leq 1\right\}$ by means of the volume form $\mu_{\mathbf{x}}=\sqrt{\operatorname{det}\left(g_{i j}\left(\mathbf{x}, \mathbf{y}_{\mathbf{x}}\right)\right)} d y_{\mathbf{x}}^{1} \wedge \cdots \wedge d y_{\mathbf{x}}^{n}$ defined on $T_{\mathbf{x}}(M) \backslash 0$. That is, $\mathbf{g}_{i j}(\mathbf{x})=\int_{B_{\mathbf{x}}} g_{i j}\left(\mathbf{x}, \mathbf{y}_{\mathbf{x}}\right) \mu_{\mathbf{x}}$. On a Landsberg manifold, both the unit balls and the volume form $\mu$ are invariant under the actions of the Berwald parallel transports. Therefore, $\nabla_{\dot{\mathbf{x}}(a)} \mu=\lim _{b \rightarrow a}\left(\tau_{\mathbf{x}(a) \mathbf{x}(b)}^{*}\left(\mu_{\mathbf{x}(b)}\right)-\mu_{\mathbf{x}(a)}\right) /(b-a)=0$. The identities $g_{i j \mid k}=0$ and $\mu_{\mid k}=0$ combined with the Leibniz rule yield the desired identity $\mathbf{g}_{i j \mid k}=0$. The proof is concluded by the above argument.

This proof can be completed also in a more direct way. In fact, equation $\mathbf{g}_{i j \mid k}=0$ implies that the parallel transports $\tau_{\mathbf{x}(a) \mathbf{x}(b)}: T_{p}(M) \rightarrow T_{q}(M)$ are isomeries with respect to the Euclidean metrics defined by $\mathbf{g}_{i j}(\mathbf{x})$ on the tangent spaces. Since the Euclidean isometries fixing the origin are linear, also these transports must be linear. Then, also the connection must be linear. For if $X_{1}(t)$ and $X_{2}(t)$ are parallel vector fields along a curve, then also $\alpha X_{1}(t)+\beta X_{2}(t)$ are parallel, for all $\alpha, \beta \in \mathbb{R}$. Thus, by Eq. 1 ,

$$
G_{r}^{i}\left(\mathbf{x}(t), \alpha X_{1}(t)+\beta X_{2}(t)\right)=\alpha G_{r}^{i}\left(\mathbf{x}(t), X_{1}(t)\right)+\beta G_{r}^{i}\left(\mathbf{x}(t), X_{2}(t)\right)
$$

holds. That is, $G_{r}^{i}(\mathbf{x}, X)=X^{s} G_{r s}^{i}(\mathbf{x})$, meaning that the connection is linear.

By this theorem, regular Berwald metrics can be characterized by the Landsberg property. Non-Berwald solutions appear just under weaker conditions. We propose investigations of Finsler metrics having Berwald parallel Finsler densities $\mu$, i.e., satisfying $\mu_{\mid k}=0$ (or, equivalently, $g^{i j} g_{k i \mid j}=0$ ). In the $2 \mathrm{D}$ case, these metrics are still Berwald. Constructions and classifications in the higher dimensions are important parts of this problem. These manifolds have the remarkable property that the integral of $\mu$ on the unit balls by means of $\mu$ defines a volume form $\omega(p)$ on the base $M^{n}$, which is also parallel (i.e., $\omega_{\mid k}=0$ holds) with respect to the Berwald connection. This means that the Berwald parallel transports are volume preserving both with respect to $\mu$ and $\omega$. In other words, there is a canonical Berwald parallel volume form, $\omega$, on the base manifold, which do not exist, a priori, on general Finsler manifolds. If they exist, they are uniquely determined upto constant factors. It is an interesting question, if the existence of a non-trivial Berwald parallel volume form $\omega$ on the base manifold implies the equation $\mu_{\mid k}=0$. Or else, there is a much wider class of Finsler manifolds which allow such canonical volume forms on the base manifold. A characterization for the latter metrics is that the elements, $\alpha$, of the Berwald holonomy group $H_{p}$ at an arbitrarily fixed point $p \in M^{n}$ are volume preserving regarding the canonical invariant measures defined on the linear space $T_{p}\left(M^{n}\right)$. This condition is equivalent to det $\alpha_{*}(y)= \pm 1$, for all $\alpha \in H_{p}$ and $y \in T_{p}\left(M^{n}\right)$. Integral geometric investigations [1,2] are also suggested on these manifolds.

Acknowledgements I am grateful to the Max Planck Institute for Mathematics in the Sciences (Leipzig) for the hospitality and excellent working conditions provided in 2007/08. My particular gratitude yields to Prof. H.-B. Rademacher for checking out the details of this article. This work was partially supported also by USA NSF Grant DMS-0604861. 


\section{References}

1. Alvarez Paiva, J.C., Fernandez, E.: Crofton formulas for projective Finsler spaces. Electr. Res. Announc. 4, 91-100 (1998)

2. Alvarez Paiva, J.C., Fernandez, E.: Fourier transforms and the Holmes-Thompson volume of Finsler manifolds. Math. Res. Not. 19, 1031-1042 (1999)

3. Asanov, G.S.: Finsleroid-Finsler space with Berwald and Landsberg conditions. arXiv: math.DG/0603472 (2006)

4. Asanov, G.S.: Finsleroid-Finsler space and geodesic spray coefficients. Publ. Math. Debrecen 71, 397-412 (2007)

5. Bao, D.: Unicorns in Finsler geometry. Preprint (2006)

6. Bao, D.: On two curvature-driven problems in Riemann-Finsler geometry. Adv. Stud. Pure Math. (2007) (to appear)

7. Bao, D., Chern, S.S., Shen, Z.: An introduction to Riemann-Finsler Geometry. Graduate Texts in Mathematics, vol. 200. Springer (2000)

8. Bryant, R.: Announcement on several occasions since 2002

9. Landsberg, G.: Über die Totalkrümmung. Jahresber. Deutsch. Math.-Verein. 16, 36-46 (1907)

10. Rund, H.: The Differential Geometry of Finsler Spaces. Springer-Verlag (1959)

11. Shen, Z.: On Landsberg $(\alpha, \beta)$-metrics. Preprint (2006)

12. Szabó, Z.I.: Positive definite Berwald spaces (Structure theorems). Tensor (N.S.) 35, 25-39 (1981)

13. Szabó, Z.I.: Berwald metrics constructed by Chevalley's polynomials. arXiv:math.DG/0601522 (2006) 